\title{
Oumuamua (A/2017U1) - A Confirmation of Links between Galactic Planetary Systems
}

\author{
N. Chandra Wickramasinghe $e^{1,2,3}$, Edward J. Steele ${ }^{4,2}$, Daryl. H. Wallis ${ }^{1}$, Robert Temple ${ }^{5}$, Gensuke \\ Tokoro $^{2,3}$, Janaki T. Wickramasinghe ${ }^{1}$ \\ ${ }^{1}$ Buckingham Centre for Astrobiology, University of Buckingham, UK \\ ${ }^{2}$ Centre for Astrobiology, University of Ruhuna, Matara, Sri Lanka \\ ${ }^{3}$ Institute for the Study of Panspermia and Astrobiology, Gifu, Japan \\ ${ }^{4} \mathrm{CY}$ O'Connor ERADE Village Foundation, Piara Waters, WA, Australia \\ ${ }^{5}$ The History of Chinese Culture Foundation, Conway Hall, London, UK \\ Email: ncwick@gmail.com
}

\begin{abstract}
The detection of an extra-solar asteroid/comet/bolide reaching the inner solar system in a hyperbolic orbit confirms the existence of a continuing material link with alien planetary systems. The Aristotelean view that the Earth and life upon are isolated from the external universe is now further challenged.
\end{abstract}

Keywords: Comets, planets, life

\section{Introduction}

Recent observations from the Kepler Mission have revealed that planetary systems similar to our solar system are exceedingly commonplace in the galaxy. The total tally of habitable exoplanets has been given as upwards of 100 billion (Kopperapu et al., 2013) [1]. Since comets and asteroids form an integral component of our solar system it would be reasonable to infer that they are also common in other planetary systems. The detection of such exocomets is, however, not easy and only in one case, the Kepler star KIC2542116, is there direct evidence of transiting comets (Rappaport et al., 2017) [2].

\section{Long-period Comets}

Comets belong to the family of minor bodies of the solar system that include asteroids, meteoroids, bodies in the Kuiper Belt and the Oort cloud, small planetary satellites and Trans-Neptunian Objects, including the planetoid Sedna. Some of these bodies have been only minimally altered from their original state in the nebula from which the solar system condensed. Comets have orbital periods ranging from a few years to a few thousand years. In general the short period comets have aphelia in the Kuiper Belt and the longer period comets originate in the Oort cloud which surrounds the solar system in a spherical shell between 2000 and $100000 \mathrm{AU}$ (1AU=Earth's mean distance from the sun).

Every year a few long-period comets make their first approach to the inner solar system in elliptic orbits of high eccentricity from the Oort cloud. Recent studies have shown that the total number of long-period comets in the inner solar system is far higher than was hitherto thought, perhaps by as much as a factor of 10 (Bauer et al., 2017)[3].

\section{Exo-comets}

There is now little doubt that a significant fraction (perhaps 30\%) of long-period comets that approach perihelion actually leaves the solar system in hyperbolic orbits (Hughes, 1991) [4]. Comets that are initially in bound elliptical orbits $(\mathrm{e}<1)$ could be deflected into hyperbolic orbits due to the effect of gravitational perturbation by Jupiter. It is this same process that caused the expulsion of the man-made spacecraft Voyager 1 and 2 into interstellar space. Because the solar system produces a steady exodus of comets the principle of mediocrity requires other similar planetary systems to do likewise. 


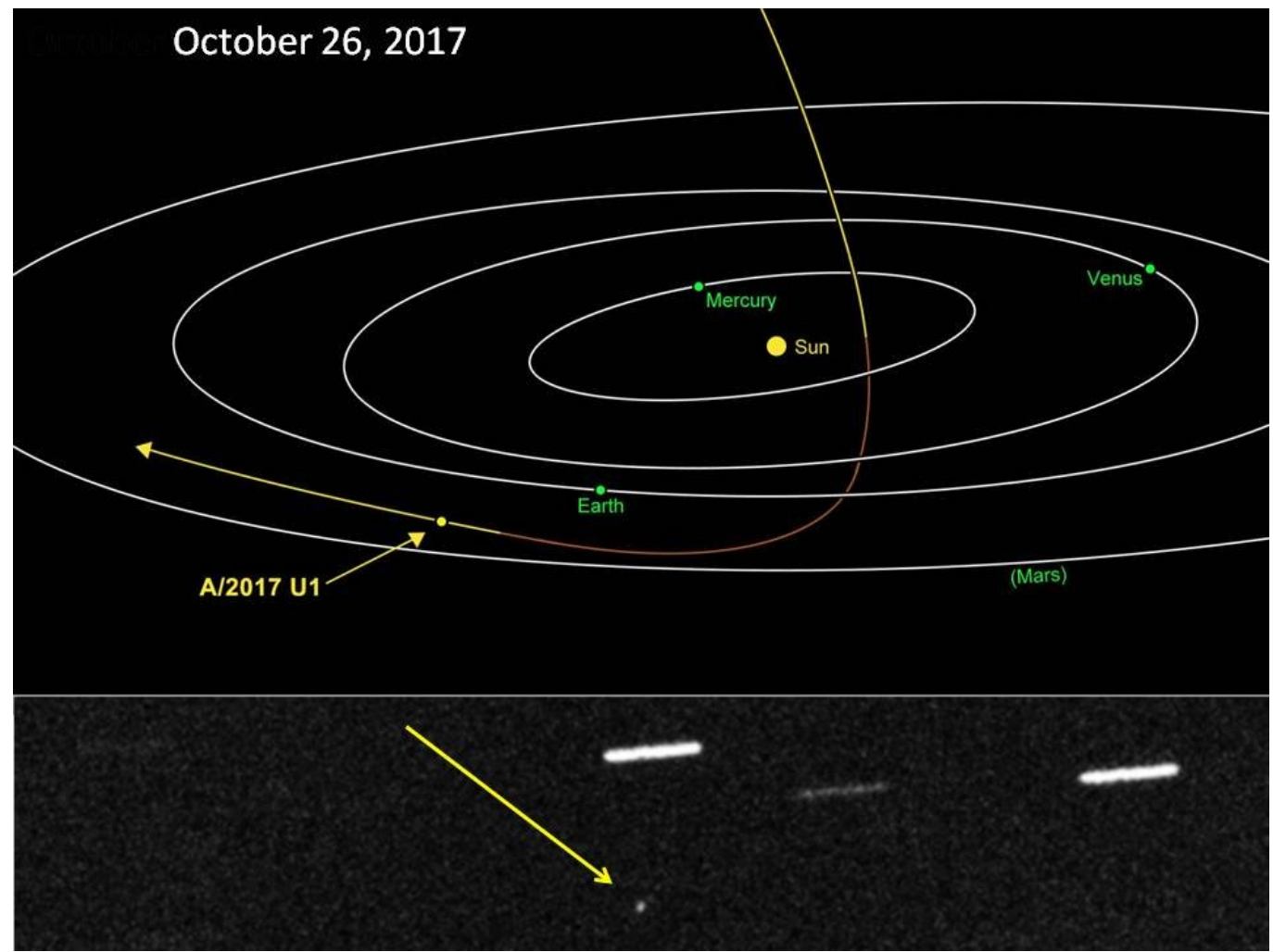

Figure 1. A/2017 orbit and detection image (below) on October 26, 2017 (Adapted from images Courtesy of NASA and Wikepedia)

If we assume that each one of a 100 billion planetary systems in the galactic disc loses 10 comets per year in hyperbolic orbits, the rate of injection of comets into the ISM is:

$d N / d t \approx 10^{11} \mathrm{x} 10=10^{12} \mathrm{yr}^{-1}$

and in a turn-over time (star formation timescale) of the ISM of $\sim 3 \times 10^{9}$ years the steady state value of $\mathrm{N}$ is

$\mathrm{N} \cong 10^{12} \times 3 \times 10^{9}=3 \times 10^{21}$

and the number density of interstellar comets is then

$\rho \approx 3 \times 10^{21} / 10^{2}=3 \times 10^{19} \mathrm{kpc}^{-3}$

taking $\sim 10^{2} \mathrm{kpc}^{3}$ to be the volume of the galactic disc. The mean separation between such comets will be $\sim \rho^{-1 / 3} \sim 10^{15} \mathrm{~cm}$, a distance close to the outer radius of the Kuiper belt of solar system comets. The expectation is therefore that hyperbolic comets must be present in our solar system at all times.

\section{The First Detection of an Exocomet in the Solar System}

After many frustrating years of searching for such hyperbolic comets the recent discovery of a hyperbolic comet/asteroid by Meech et al. (2017) [5] came as a welcome relief. The discovery on October $19^{\text {th }} 2007$ of the object designated A/2017U1 was soon confirmed to be extrasolar in origin coming in a hyperbolic orbit of high inclination to the ecliptic, with perihelion distance $q=0.25 \mathrm{AU}$ and orbital eccentricity $\mathrm{e}=1.19$. Its classification as a comet was rejected on the grounds that there was no evidence of outgassing, a dust tail or a coma, normally expected from solar system comets that approach as close as $0.25 \mathrm{AU}$ to the sun. In the event the classification as an asteroid was evidently preferred with a designation thus given as A/2017U1. We do not consider this classification to be more than a working hypothesis, however.

If material of normal cometary composition is exposed to interstellar radiation for millions of years it is more than likely that its surface would have undergone significant chemical alteration. With a reddish 
colour and a reflectivity spectrum not dissimilar to that of comet $67 \mathrm{P} / \mathrm{C}-\mathrm{G}$ a cometary regolith denuded of volatiles would be the most reasonable explanation of the surface properties of A/2017U1 [5]. Such a surface could be similar to that of a "burnt out" comet after many perihelion passages that is dominated by highly refractory organic polymers as discussed by Hoyle and Wickramasinghe (1986)[6] and Napier, Wickramasinghe and Wickramasinghe (2004)[7]. Outgassing from such a comet would be choked off until an impact occurred with a meteoroid in the zodiacal cloud. If subsurface microbiology still resided deep under the surface, it is possible that an impact might trigger metabolism and explosive outburst of gas and dust (Wickramasinghe, Hoyle \& Lloyd, 1996) [8]. Such discrete impact events may well have taken place and escaped notice before the comet came to be first detected in October 2017. Excluding such impacts, from the non-detection of a coma and dust tail, Meech et al. (2017) [5] have set an upper limit of $1.7 \mathrm{~kg} / \mathrm{s}$ of ejected gas or $10 \mathrm{~g} / \mathrm{s}$ of dust.

An interesting consideration relates to the possibility that objects such as A/2017 may serve to transfer material from the zodiacal cloud, including traces of biological material arising from the solar system's planets and comets, to distant star systems. Consider a highly porous object of effective radius $200 \mathrm{~km}$ ploughing through a distance of $0.2 \mathrm{AU}$ through the inner zodiacal dust cloud of density $3.10^{-22} \mathrm{~g}$ $\mathrm{cm}^{-3}$. The total mass of zodiacal dust encountered is then found to be $100 \mathrm{~kg}$. Even if only a small fraction of this material comes to be implanted in the porous crevices of A/2017U1, this would provide a means of transporting biological material indigenous to the solar system to other distant stellarplanetary abodes.

A highly elongated structure for A/2017U1 with a notional dimension of $180 \mathrm{~m} \times 30 \mathrm{~m} \times 30 \mathrm{~m}$ has been inferred from its highly variable light curve, assuming a geometric albedo of 0.1 (Jewitt et al., 2017) [9]. Meech et al. [5] obtain a marginally larger comet by assuming a cometary albedo of 0.04 typical of most comets in the solar system. These dimensions may be revised upwards if a much lower albedo is appropriate (see accompanying paper), but in any case the formation and stability of such a thin long oblate spheroidal structure poses a serious problem. Other ways of modelling the available light curve data could be explored, including the possibility that it is a composite object made of smaller bodies. Finally the exotic but admittedly inadmissible possibility is that A/2017U1 is the artefact of an intelligent civilization similar to our own Voyager spacecraft now hurtling through interstellar space. One way or other the discovery of our first "visitor" on a hyperbolic orbit confirms our inextricable connection with life that may exist in distant planetary systems.

\section{Concluding Remarks}

The theory that comets and cometary bodies are the means of transport for biology throughout the galaxy has been extensively discussed by Hoyle, Wickramasinghe and their many collaborators for over nearly 4 decades [10]. A rapidly growing body of evidence from within biology itself, as well as from geology, astronomy and space research, is now converging towards a vindication of the HoyleWickramasinghe theory of the origin and evolution of cosmic life (Steele et al., 2017) [11]. In a comprehensive review article Steele et al. [11] have shown that this long-resisted paradigm shift is well under way at the present time. The arrival of Oumuamua (A/2017U1) may coincide with the ushering in of a new transformative paradigm in science.

\section{References}

1. Kopparapu, R.K. et al., "Habitable zones around Main-Sequence stars: New estimates," Astrophys.J. 765, 131, 2013.

2. Rappaport, S. et al., "Likely transiting exocomets detected by Kepler," Monthly Notices of the Royal Astronomical Society, stx2735, 2017.

3. Bauer,J.M., Grav, T., Fernandez, Y.R. et al., "Debiasing the NEOWISE cryogenic missing comet populations," Astron.J, 154(2), 2017.

4. Hughes, D.W., "On hyperbolic comets, British Astronomical Association," 101(2), 1991.

5. Meech, K.J., Weryk, R., Micheli, M. et al., "Discovery and characterisation of the first known interstellar object," Nature, 2017. 
6. Hoyle, F. and Wickramasinghe, N.C., "Some predictions on the nature of comet Halley," Astrophysics and Space Science, Volume 268(1-3): 343-347, 1999.

7. W. M. Napier, J. T. Wickramasinghe, N. C. Wickramasinghe. "Extreme albedo comets and the impact hazard," Monthly Notices of the Royal Astronomical Society, Vol. 355, No. 1: 191-195, 2004.

8. Wickramasinghe, N.C., Hoyle, F. and Lloyd, D., (1996). Eruptions of Comet Hale-Bopp at 6.5AU, Astrophys. Sp. Sci. 240: 161-165, 1996

9. Jewitt, D., Luu, J., Rajgopal, J. et al., "Interstellar Interloper II/2017U1: Observation from the NOT and WIYN Telescopes," Astrophys J. Lett (in press), 2017.

10. Wickramasinghe, C., The search for our cosmic ancestry, World Scientific Press, Singapore, pp. 143-158, 2014.

11. Steele, E.J., Al-Mufti, S., Augustyn, K.A. et al., "Cause of Cambrian Explosion - Terrestrial or Cosmic?" Progress in Biophysics and Molecular Biology (in press), 2017. 